\title{
Drugs in development for treatment of patients with cancer-related anorexia and cachexia syndrome
}

This article was published in the following Dove Press journal:

Drug Design, Development and Therapy

10 August 2013

Number of times this article has been viewed

\author{
Giovanni Mantovani' \\ Clelia Madeddu' \\ Antonio Macciò ${ }^{2}$ \\ 'Department of Medical Oncology, \\ University of Cagliari, Cagliari, \\ Italy; ${ }^{2}$ Department of Gynecologic \\ Oncology, A. Businco Hospital, \\ Regional Referral Center for Cancer \\ Disease, Cagliari, Italy
}

\begin{abstract}
Cancer-related anorexia and cachexia syndrome ACD is a complex multifactorial condition, with loss of lean body mass, chronic inflamm on, severe etabolic derangements, reduced food intake, reduced physical activity, ar poor uality 9 life as key symptoms. Cachexia recognizes different phases or stages, $n$, ing from pre dia through overt cachexia to advanced or refractory cachexia. The purnoso th eview is to describe currently effective approaches for the treatment of cachexia. oving fo ard to ugs and treatments already shown

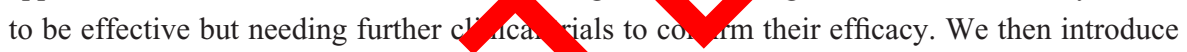
novel promising investigational druos and app aches which, based on a strong rationale from the most recent data on the mo cular targets/pathways driving the pathophysiology of cachexia, need to be tested either in cur ntly ongor or appropriate future clinical trials to confirm their clinical potential. Although di rent dry and treatments have been tested, we can speculate that a single therapy and be compretely successful. Indeed, considering the complex clinical picture and the multif. tor pangenesis of CACS, we believe that its clinical management requires a $y$ cith ciplit y and multitargeted approach. In our opinion, appropriate treatment for cack xia sh ld targ the following conditions: inflammatory status, oxidative stress, nutry ona riders, indscle catabolism, immunosuppression, quality of life, and above all, gue. A co rehensive list of the most interesting and effective multitargeted treatments is repo $d$ and discussed, with the aim of suggesting the most promising with regard to clinical outcomo critical issue is that of testing therapies at the earliest stages of cachexia, possibly at the precachexia stage, with the aim of preventing or delaying the development of overt cachexia and ereby obtaining the best possible clinical outcome for patients.

-ywords: proinflammatory cytokines, nutritional status, metabolic derangements, quality of life, cachexia staging, multimodal therapy
\end{abstract}

\section{Introduction}

Cancer-related anorexia and cachexia syndrome (CACS) is a debilitating clinical condition that affects the course of several chronic diseases, including chronic heart failure, chronic obstructive pulmonary disease, chronic kidney disease, and especially cancer. During its progression, cancer induces changes in the host immune system and energy metabolism that affect the clinical status of the patient so profoundly that it can result in death. ${ }^{1}$ The following symptoms are associated with these events and involve various organs and systems: anorexia, nausea, weight loss (with a reduction in lean body mass and adipose tissue), increased energy metabolism (with changes in glucose, lipid, and protein metabolism), immunosuppression, and fatigue. All these symptoms ultimately result in the clinical picture of CACS, which, unless counteracted,
Correspondence: Giovanni Mantovan Policlinico Universitario, SS 554, Km 4500, 09042 Monserrato,

Cagliari, Italy

Tel +39070 6754250

Fax +39070675 4250

Email mantovan@medicina.unica.it 
has a negative impact on quality of life for patients. ${ }^{2} \mathrm{~A}$ recent consensus defined cachexia as "a complex metabolic syndrome associated with an underlying inflammatory disease and characterized by the loss of muscle with or without loss of fat mass". ${ }^{3}$ The pathophysiology of cachexia is common, at least in part, in the different diseases, and represents the main background of cachexia symptoms. In this review, we focus on CACS, the mechanisms of which are shared by chronic illnesses.

It is well established that proinflammatory cytokines, including interleukin (IL)-1, IL-6, and tumor necrosis factor (TNF)- $\alpha$, which are produced by the activated immune system and by tumor cells, are involved in the pathophysiology of CACS and the associated metabolic changes. ${ }^{4}$ It may be hypothesized that the synthesis and release of proinflammatory cytokines may lead to an efficient antineoplastic effect during the initial phases of neoplastic disease. However, the inability of the immune system to counteract tumor growth ultimately results in chronic cytokine activity, with irreversible effects on cell metabolism, body composition, nutritional status, and immune system efficiency. ${ }^{5}$ In turn, proinflammatory cytokines promote the synthesis of acute-phase proteins, which contribute to the pathogenesis of altered energy metabolism. ${ }^{6}$ Proinflammatory cytokines, together with tumor-derived factors, such as proteolysis-inducing fac and the recently discovered myostatin, ${ }^{7}$ also play a centr role in the pathogenesis of muscle wasting via a no the ubiquitin-proteasome proteolytic pathwa

A major clinical feature of CACS mass, leading to fatigue, impairment roc aco ity, and eventually death. ${ }^{9}$ Muscle wasting is the in ylt of multiple alterations at both the molecular nd metabolic vels, leading to a disturbance in the balan bety en protein degradation and protein synthesis, y related to enhanced ase of musch roteins as an energy source to supply the crear gy needs of patients with cachexia.

Anorexia, which is also induced by proinflammatory cytokines, ${ }^{10,11}$ is often associated with CACS, leading to reduced food intake. However, anorexia alone cannot account for the complex alterations characterizing this syndrome, thus confirming that cachexia is not just a consequence of malnutrition, but that other events are involved in its pathogenesis. $^{12}$

In this context, the finding that cancer patients in advanced stages of the disease show severe impairment of immune function, characterized by a cell-mediated immunity deficit, elevated serum levels of proinflammatory cytokines, and acute-phase proteins (fibrinogen and C-reactive protein), is very relevant ${ }^{13}$ and encompasses the chronic inflammation status typical of CACS. ${ }^{14}$ The exact time when these changes occur is difficult to establish, but they are probably due to an interaction between the tumor and host. The tumor and its continuous growth are responsible for increased energy expenditure and progressive weight loss. ${ }^{15}$ Moreover, tumor growth is accompanied by chronic activation of the immune system as it triggers a response to counteract the tumor. The immune response is also energetically costly $(25 \%-30 \%$ of the basal metabolic rate, ie, $1750-2080 \mathrm{~kJ} /$ day). ${ }^{16}$

From the evidence discussed above, it is intuitive that the clinical management of CACS is complex and requires a multidisciplinary and multipharmacological approach. ${ }^{17,18}$ Appropriate treatment of $C$ CS s uld include drugs that address the following nditions: nflammatory state, nutritional disorder etabo deran ements, immunological defects, poor ,uality life, $d$, in particular, fatigue. Accordingly cat for CA should include as primary endpoints followin. ables, which were recently identified as key ih achexia: ${ }^{19}$ an increase in lean body mass an runctional act ty (grip strength, physical activity mea$s$ red by either the six-minute walk test, arm band device, o. hree-ste treadmill test); a decrease in resting energy exper.e; and improvement of fatigue. Moreover, the on variables should be included as secondary endpoints: increased appetite, improved quality of life assessed by EORTC-QLQ-C30, and a decrease in proinflammatory cytokines (IL-6, and TNF- $\alpha$ ). In fact, only full knowledge of the pathophysiology of CACS will enable identification of the most appropriate drugs to counteract the constitutive symptoms. A comprehensive summary of potentially available drugs for CACS is shown in Table 1.

\section{Treatment of CACS Progestagens}

Progestagens were the first agents used for the treatment of CACS and are currently the only agents approved in Europe for its treatment. An extensive amount of literature is available on megestrol acetate and medroxyprogesterone acetate (MPA) for the treatment of patients with cancer. ${ }^{20}$ Megestrol acetate and MPA are equivalent in terms of effectiveness in the treatment of CACS. However, megestrol acetate has been more widely investigated for its effect on cachexia ${ }^{21}$ than MPA. ${ }^{20}$ The positive effects of megestrol acetate on weight and well-being have been observed at oral doses in the range of 160-1600 mg/day. However, because megestrol acetate may be associated with severe dose-related adverse 
Table I Comprehensive summary of drugs potentially available for cachexia in cancer patients

Ineffective treatments

- Cyproheptadine

- Hydrazine

- Metoclopramide

- Pentoxifylline

Drugs commonly used

- Progestagens

- Megestrol acetate

- Medroxyprogesterone acetate

- Corticosteroids

- Anabolic agents (oxandrolone)

Drugs with a strong rationale that failed or showed equivocal results in clinical trials

- Omega-3 fatty acids

- Cannabinoids (dronabinol)

- Bortezomib

Drugs with confirmed clinical results

- COX-2 inhibitors

- Thalidomide

- Melatonin

- Insulin

- Branch-chained amino acids

Investigational drugs with clinical effectiveness to be confirmed

- Ghrelin and ghrelin mimetics

- Melanocortin antagonists

- Drugs targeting inflammatory cytokines

- Selective androgen receptor modulators

- Myostatin inhibitors

- $\beta$-adrenoceptor agonists

Investigational new drugs registered at ClinicalTrials.gov Multimodal therapy

effects, starting treatment at a low dose titrating the dose upwards according inical re onse is recommended. ${ }^{22} \mathrm{MPA}$ has been used at dos in the range of 300-4000 mg/day. In a placeb controlled stus by Simons et al, ${ }^{23}$ a significant improver nt in spetite and body weight was achieved using an do of $\mathrm{M} A$ in the range of 500-1000 mg/day. oreoy;, a sy natic review of MPA for the treatment of $4 C$ coum nat there were no significant differences betwee, igh and low doses. ${ }^{24}$ Therefore, an MPA dose of 500-1000 mgday orally can be recommended in clinical practice. Both megestrol acetate and MPA may have adverse effects, including an increased risk of thromboembolic events, peripheral edema, breakthrough bleeding, hyperglycemia, hypertension, and Cushing's syndrome. Recently, an oral suspension formulation of megestrol acetate was developed using nanocrystal technology. Preclinical pharmacokinetic data suggest that this formulation of megestrol acetate can produce a more rapid clinical response by rapidly increasing plasma megestrol acetate levels. ${ }^{25}$ The US Food and Drug Administration (FDA) approved the oral suspension for the treatment of acquired immune deficiency syndrome-related cachexia, and this drug is currently under evaluation for approval to treat CACS associated with other conditions.

\section{Corticosteroids}

Several randomized, placebo-controlled studies have shown that corticosteroids achieve a limited (up to one month) improvement in appetite, food intake, nausea, and feeling of well-being. However, none of these studies showed an increase in body weight. ${ }^{26-30}$ The rapid beneficial effect of corticosteroids on mood and behavior significantly improves quality of life. The mechanism of action of corticosteroids in CACS is not well understood, although inhibition of prostaglandin activity and ppre ion of IL-1 and TNF- $\alpha$ production and release aro most wo recognized targets. ${ }^{22}$ The specific drug, de, and ute of dministration of corticosteroids are st well stabl de however, low doses, equivalent to ess $1 \mathrm{mg} / \mathrm{g}$ of prednisone, are recommended in inical pr. Further, because of their well known adverse fects, short-term (no more than 1-2 months) or monating use fose agents is recommended in the r anagemer of CACS.

Antaric agents

lic androgens are synthetic derivatives of testosterone, with a greater anabolic effect and less androgenic activity than testosterone. Studies on the use of these anabolic agents in cachectic patients have been limited largely to patients with chronic obstructive pulmonary disease and human immunodeficiency virus/acquired immune deficiency syndrome, in whom positive effects on body weight, lean body mass, and several functional parameters were observed. However, few studies have been carried out to date in patients with CACS.

Recently, a prospective, randomized Phase III trial compared the effects of oxandrolone $10 \mathrm{mg}$ twice daily and megestrol acetate $800 \mathrm{mg}$ daily on weight, body composition, and quality of life in 155 adult patients with solid tumors and weight loss while receiving chemotherapy. This study showed that patients treated with oxandrolone experienced an increase in lean body mass, a reduction in fat mass, and fewer self-reported anorectic symptoms. ${ }^{31}$ The side effects of these agents include elevated transaminase levels (especially with nandrolone), decreased high-density lipoprotein levels, interactions with oral anticoagulants, oral hypoglycemics, and adrenal steroids, and hypogonadism (with decreased systemic testosterone levels). Oxandrolone is administered 
orally (at approved dose concentrations of 5-20 mg/day) and has a better safety profile and less potential for hepatic toxicity and virilizing effects than other androgens. This agent is well tolerated in women. ${ }^{32}$

\section{Drugs with confirmed clinical results Nonsteroidal anti-inflammatory drugs} COX-2 selective inhibitors

The development of selective COX-2 inhibitors has resulted in safer modulation of cancer-associated inflammation, and these agents could help alleviate or control CACS. Moreover, the selective COX-2 inhibitors have shown potent inhibitory and preventive effects on tumor growth in animal models; therefore, their antineoplastic activity may contribute to their ability to counteract cachexia. In particular, use of celecoxib, a selective COX-2 inhibitor, has been investigated. Lai et $\mathrm{al}^{33}$ randomized 11 cachectic patients with head and neck or gastrointestinal cancer to receive celecoxib $200 \mathrm{mg}$ twice daily or placebo for three weeks. The patients on celecoxib reported good compliance and no adverse events were observed. Patients on celecoxib also showed a nonsignificant increase in body weight (mean change $+1.0 \mathrm{~kg}$ versus $-1.3 \mathrm{~kg}$ in the placebo group) and a significant increase in quality of life. A recent nonrandomized, prospective Phase II study investigated celecoxib $300 \mathrm{mg}$ /day for four mon in 24 patients with advanced cancer. ${ }^{34}$ The results indicato a significant decrease in levels of the proinf am atory cytokine, TNF- $\alpha$, and a significant increas in lea hody mass. In addition, significant improveme $i_{\mathrm{S}} \mathrm{wo}$ observed in quality of life, performance statu " asgow P1 nostic Score, and grip strength. Patient ompliano was good and no severe toxicities were obs ved. Qn the basis of these results, celecoxib can be in yde as a component in the combined treatment a $\operatorname{targ}$ the inflammatory environment of $\mathrm{CA}^{\prime}$

A randomized $P$ a triarassessing the feasibility of recruitment and retentio of patients with advanced nonsmall cell lung cancer (NSCLC) undertaking a 12-week multimodal intervention of celecoxib, oral nutritional supplements, and physical exercise is due for completion by December 2014. ${ }^{35}$

\section{Thalidomide}

Thalidomide has complex immunomodulatory and antiinflammatory properties. It downregulates the production of TNF- $\alpha$ and other proinflammatory cytokines, inhibits transcription factor nuclear factor (NF-kB), downregulates COX-2, and inhibits angiogenesis. Therefore, thalidomide is a novel and rational treatment approach for CACS. In a randomized, placebo-controlled trial, thalidomide was found to be well tolerated and effective in slowing weight loss and improving arm muscle mass and physical function in 33 patients with advanced pancreatic cancer and CACS. ${ }^{36}$ Recently, a meta-analysis was performed to assess whether thalidomide is an effective treatment for $\mathrm{CACS},{ }^{37}$ and the authors concluded that there is inadequate evidence to recommend the use of this drug in clinical practice. Further large, well conducted, randomized controlled trials are needed to assess properly the true benefits of thalidomide alone and in combination in CSCS.

Lenalidomide Revlimid $^{\mathrm{TM}}$, Celgene Corporation, Summit, NJ, USA) is a derivative of thalidomide now approved by the FDA for th Treant of myelodysplastic syndromes. A randomize multicent Phase II trial is presently underway ass sing th effica $y$ of lenalidomide in enhancing lean b ay ma and g strength in patients with advanced cap

\section{Melatomin}

De ando et al yerformed a randomized, double-blind, 2 -day trial f melatonin $20 \mathrm{mg}$ versus placebo in patients w h advang $d$ lung or gastrointestinal cancer and a history of thoss $\geq 5 \%$. Assessments included weight, sympon the Edmonton Symptom Assessment Scale, and quality of life using the Functional Assessment of Anorexia/ Cachexia Therapy questionnaire. After interim analysis of 48 patients, the study was closed because of futility. There were no significant differences between the treatment groups with regard to appetite or other symptoms, weight, Functional Assessment of Anorexia/Cachexia Therapy score, toxicity, or survival from baseline to day 28. Therefore, oral melatonin $20 \mathrm{mg}$ at night did not improve appetite, weight, or quality of life compared with placebo.

\section{Investigational drugs with clinical effectiveness to be confirmed}

\section{Ghrelin and ghrelin mimetics}

Ghrelin is a 28-amino acid peptide produced by the P/D1 cells of the stomach, and stimulates secretion of growth hormone (GH, through the GH secretagogue-1a [GHS-1a] receptor), promotes food intake (through the orexigenic neuropeptide $\mathrm{Y}$ system), and decreases sympathetic nerve activity. Based on animal and short-term human trials, the evidence for use of ghrelin and GHS-R agonists in the treatment of CACS seems promising. Synthetic human ghrelin has been shown to improve muscle wasting and functional capacity 
in patients with cardiopulmonary-associated cachexia. ${ }^{39}$ Single-dose intravenous administration of ghrelin to cancer patients with cachexia did not show univocal efficacy in increasing food intake. In a randomized placebo-controlled trial, RC-1291 (anamorelin, an orally active small molecule GHS-R agonist) was administered to 81 patients with a variety of cancers (predominantly lung cancer) over a 12-week period. RC-1291 improved total body mass and there was a trend towards increased lean body mass, but quality of life was unchanged. ${ }^{40}$ More recently, anamorelin was shown to increase body weight and anabolic hormone levels in healthy volunteers. This drug was also investigated as a treatment for CACS in 16 patients with different types of cancer, and achieved a significant increase in body weight and improvement in patient-reported symptoms, including appetite, compared with placebo. ${ }^{41}$ However, these were small Phase I and Phase II trials, so their results should be interpreted with caution. A randomized, double-blind, placebo-controlled Phase III trial is presently enrolling up to 477 patients with NSCLC and CACS to measure lean body mass and muscle strength. This trial, sponsored by Helsinn Therapeutics (Bridgewater, NJ, USA), started recruiting in 2011 and is expected to be completed by 2014 (see Table 2). A caveat to the use of ghrelin agonists for treating CACS is the potential for stimulating tumor growth. Ghrelin and its receptor expressed in many tumor cells and may contribute to tum progression. Although no clinical study has $\mathrm{pos}$ an increased tumor incidence with administration of ghre $h$. the studies to date have been short-term only hero , further randomized, controlled studies are wa ted befor the use of ghrelin can be translated into clinical pi tice.

AEZS-130 is an oral pept omimetic grow hormone secretagogue developed by tern Lentaris Inc (Quebec, Canada), and was shor to b vell t crated in healthy subjects. ${ }^{35}$ A proof-conc t stud, patients with cancer and cachexia was phe cosmen 2011.

\section{Melanocortin antagonist.}

Among the appetite stimulants, a promising approach is targeting of the melanocortin-4 receptor. Interesting results were observed in colon-26 tumor-bearing mice, ${ }^{42}$ and clinical studies of this agent are planned..$^{35}$

\section{Drugs targeting inflammatory cytokines}

The most effective anti-inflammatory drugs have been those targeting TNF- $\alpha$ and IL-6. A humanized monoclonal anti-IL-6 antibody, ALD518 (Alder Biopharmaceuticals Inc, Bothell, WA, USA), may also benefit patients with cancer-associated cachexia because its administration increases hemoglobin levels and prevents reduction in lean body mass in those with advanced NSCLC. ${ }^{43}$

Greater benefits may be conferred when TNF- $\alpha$ and IL-6 are targeted simultaneously. OHR Pharmaceutical Inc (New York, NY, USA) have developed the broad-spectrum peptide nucleic acid immune modulator drug, OHR/AVR118, which targets both TNF- $\alpha$ and IL-6 and maintains immune homeostasis. In a Phase II study, eight of 21 enrolled patients with advanced cancer completed the study, and showed an improvement in anorexia, dyspepsia, strength (assessed by grip strength), and depression. ${ }^{44} \mathrm{~A}$ Phase IIb trial is currently assessing the efficacy of OHR/AVR118 in improving appetite and enhancing body mass, lean mass, strength (assessed by grip strength), and quality nten patients with recurrent or advanced cancer and $\mathrm{w}$ expected be completed before the end of Novembe 2011 .

A humanize anti-I/ 6 anı dy (BMS-945429) was shown to be afe well torated during early clinical studies in rients wh $\mathrm{N}^{\circ}$ CL, with treatment improving lung symptom, and reversing fatigue, with a trend towards a crouse in loss $f$ lean body mass. ${ }^{45}$ These findings are nsistent $r$ th the results of a Phase II trial that assessed so metinib an inhibitor of MAPK1 and IL-6 secretion) in 20 rents with cholangiocarcinoma. ${ }^{46}$ Overall, $84 \%$ 1. tients in this trial showed a mean muscle gain of $2.3 \mathrm{~kg} .{ }^{46}$

\section{Selective androgen receptor modulators}

Due to the lack of selectivity of anabolic androgens, a need for more selective anabolic agents has emerged, resulting in the development of nonsteroidal selective androgen receptor modulators (SARMs). These agents have the potential to elicit beneficial anabolic effects in a tissue-selective manner, while avoiding many of the side effects observed with steroidal agents. The first nonsteroidal SARM was reported in 1998, and many of the major pharmaceutical companies have disclosed the specific chemical structure of different SARMs. Currently, the agent furthest into clinical development is enobosarm (GTx Inc, Memphis, TN, USA) for the potential prevention and treatment of muscle wasting in patients with cancer. In a Phase IIb clinical trial (ClinicalTrials. gov, NCT00467844) in patients with CACS, treatment with enobosarm significantly improved lean body mass, physical performance, and quality of life compared with baseline. Currently, two Phase III trials (ClinicalTrials.gov, NCT01355484 and NCT01355497) are recruiting patients with NSCLC to assess the effects of enobosarm on muscle wasting. Further, 


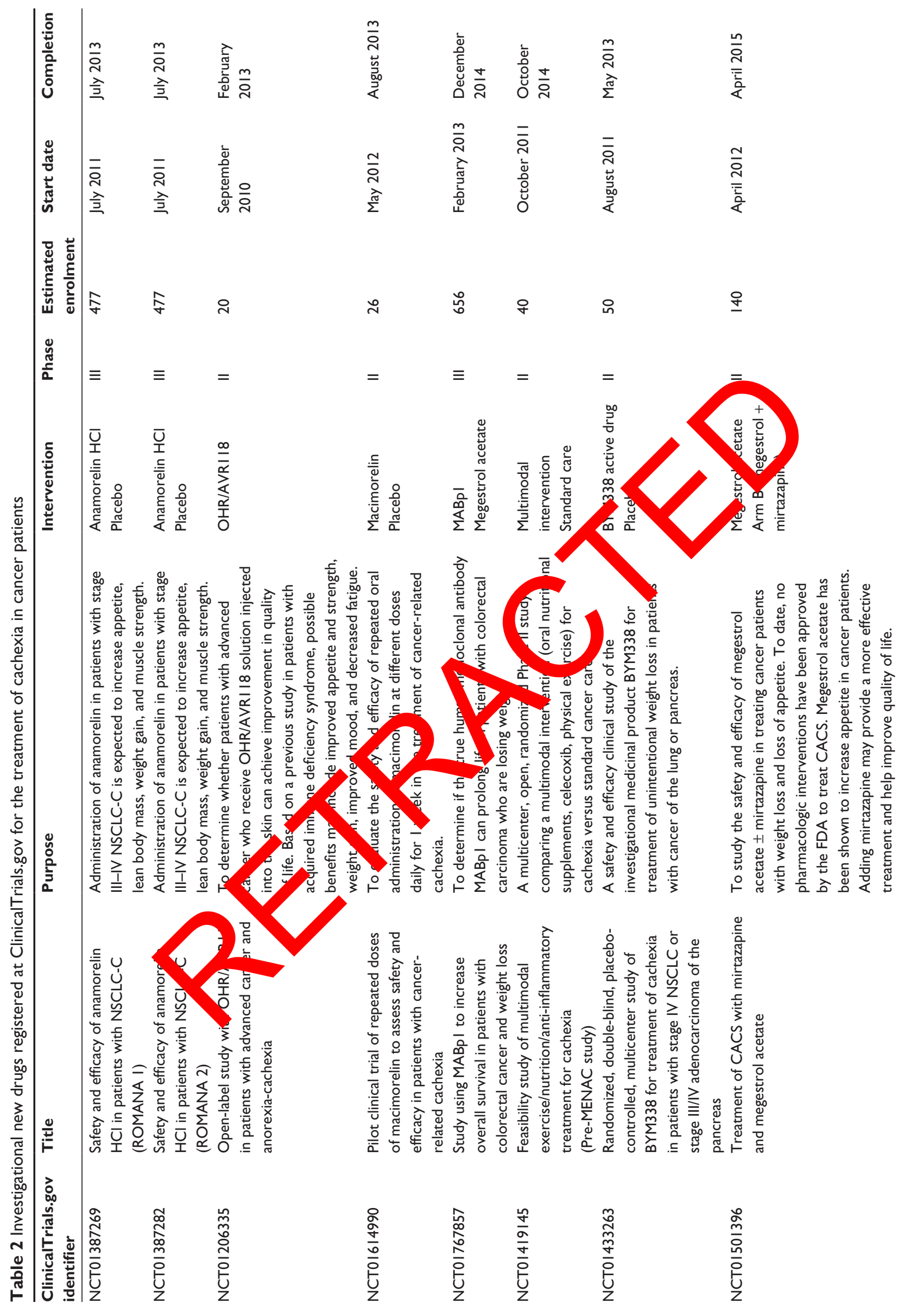




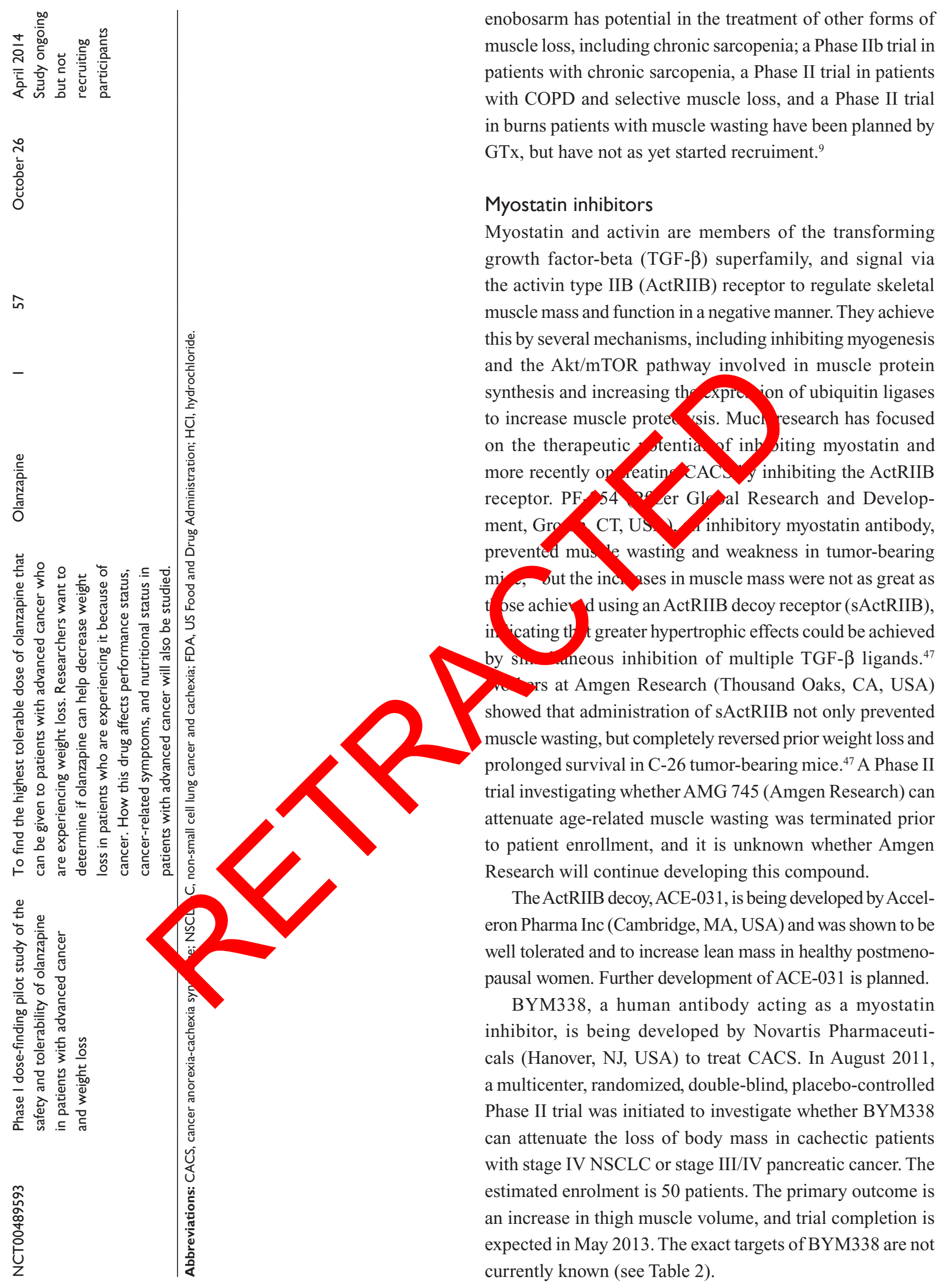


LY2495655 is another antimyostatin monoclonal antibody. A multicenter, randomized, double-blind, placebocontrolled Phase II trial in patients with locally advanced or metastatic pancreatic cancer will investigate two different doses of LY2495655 in combination with gemcitabine. ${ }^{48}$ Overall survival is the primary outcome of this study, with secondary endpoints including muscle mass and physical performance.

\section{$\beta$-adrenoceptor agonists}

The hypertrophic effects of $\beta 2$-adrenoceptor agonists, such as formoterol, in cachectic tumor-bearing rodents are well established. ${ }^{49}$ APD209 (Acacia Pharma Ltd, Harston Mill, UK) is an oral fixed-dose combination of formoterol and megestrol, and a Phase IIa study investigating the effects of eight weeks of treatment in 13 patients with CACS was recently completed. Six of the seven patients who completed the study demonstrated improved muscle size and strength, and three patients had increased levels of daily physical activity. Few patients reported side effects, such as muscle tremor or tachycardia. Acacia Pharma is currently planning larger randomized studies of this agent.

MT-102 (PsiOxus Therapeutics Ltd, Billericay, UK) is an anabolic/catabolic transforming agent with properties including nonspecific $\beta 1$-adrenergic and $\beta 2$-adrenergic rece antagonism, intrinsic sympathomimetic activity, and 5-HT receptor antagonism. MT-102 increased food in an body mass, fat and lean muscle mass, physical acti cy leve a. and survival time in cachectic tumor-bearing r $7{ }^{50} \mathrm{~A}$ iticenter, randomized, double-blind Phase II tria' s initiate in April 2011 to investigate whether up to 16 weeks treatment with MT-102 would improve the $r$ of change $\mathrm{d}$ body mass compared with placebo in at ast $12 /$ patients with stage III and IV NSCLC or colo ql ca er an ACS. ${ }^{51}$ The estimated study comple on dat was A.ast 2012, and enrolled patients who comp d d ceatment period and still taking randomizeo, ouble-blind trial medication were offered the opportunity to Join in a subsequent trial with a separate primary endpoint.

\section{Investigational new drugs registered at Clin Trials.gov}

The investigational new drugs registered at ClinicalTrials. gov for the treatment of CACS are shown in Table 2.

\section{Multimodal therapy}

To date, studies on CACS therapy using various single interventions have had limited success. The main features of cachexia, ie, progressive loss of muscle mass and function, are minimally influenced by the nutritional and pharmacological tools currently available. The lack of efficacy of monotherapy is due to the multifactorial pathogenesis of cachexia. Therefore, a combination of dietary, nutritional, and pharmacological approaches targeting the main factors contributing to cachexia may be able to normalize the metabolic milieu and thus reverse cachexia-related symptoms that impact quality of life for patients. ${ }^{18}$ Several studies in the last decade have investigated the combination of megestrol acetate with other drugs.

The combination of megestrol acetate with tetrahydrocannabinol ${ }^{52}$ and with eicosapentaenoic acid $^{53}$ did not provide any benefits compared with use of megestrol acetate alone. However, megestrol acetate ith in profen was more effective than either drug usec tone. ${ }^{54}$

An interesting pi $/$ study erforn $d$ by Cerchietti et al ${ }^{55}$ demonstrated th effica of ombined approach in a homogeneour rou $_{1} 15$ pati ts with lung adenocarcinoma and evide of sys ni Immune metabolic syndrome, which was den by the authors as a distressing systemic sy arne charac ized by weight loss, anorexia, fatigue, n rformang status $\leq 2$, and an acute-phase protein response. T. multita eted approach consisted of MPA $500 \mathrm{mg}$ twice daily celecoxib $200 \mathrm{mg}$ twice daily as well as oral food ar mentation for six weeks. This combined treatment significantly improved the rate of change in body weight, nausea, early satiety, fatigue, appetite, and performance status. In a subsequent study, the same authors ${ }^{56}$ randomized 22 patients with advanced lung cancer and systemic immune metabolic syndrome to receive either fish oil $2 \mathrm{~g}$ three times daily plus placebo or fish oil $2 \mathrm{~g}$ times daily plus celecoxib $200 \mathrm{mg}$ twice daily. All patients in both groups received oral food supplementation. After six weeks of treatment, patients in both arms showed a significantly increased appetite, improvement in fatigue, and lower C-reactive protein levels compared with baseline. Patients in the celecoxib group showed improved body weight and muscle strength compared with baseline and a significantly lower C-reactive protein level and greater muscle strength and body weight than patients who received placebo.

Lundholm et $\mathrm{al}^{57}$ assessed whether a combined approach, including daily insulin plus anti-inflammatory treatment (indomethacin), rHuEPO, and specialized nutritional care (oral supplements plus home parenteral nutrition), attenuated the progression of cancer-related cachexia and improved metabolism and physical functioning in 138 unselected patients with advanced gastrointestinal cancer. 
The combined treatment significantly stimulated carbohydrate intake, decreased serum-free fatty acids, and increased whole body fat, whereas fat free lean tissue mass was unaffected. Moreover, the combined treatment improved metabolic efficiency during exercise, but did not increase maximum capacity during exertion and spontaneous physical activity.

The safety and efficacy of a combined approach was also tested by Mantovani et al in controlled clinical studies of cachectic patients with advanced tumors at different anatomical sites. First, a Phase II study, ${ }^{58}$ carried out according to a Simon's two-stage design in a population of 39 patients with advanced cancer and CACS, showed that a combined approach, which included antioxidants + L-carnitine + eicosapentaenoic acid supplementation + celecoxib + MPA, was both safe and effective in increasing body weight and lean body mass, decreasing proinflammatory cytokines, improving quality of life parameters, and ameliorating symptoms of fatigue.

On the basis of these positive results, Mantovani et al carried out a randomized Phase III trial in 332 patients with CACS to establish the most effective and safest treatment for CACS with regard to the primary endpoints of increased lean body mass, decreased resting energy expenditure, and improvement of fatigue, and included several significant ondary endpoints, ie, improvement in appetite, improveme in quality of life, increase in grip strength, decrease 10 sgow Prognostic Score, and decrease in proinflamm cory cy skine levels. ${ }^{59}$ All patients were given basic treatr ont polyphenols plus antioxidant agents, ie, $\alpha$-lipo cid, carbo vsteine, and vitamins $\mathrm{A}, \mathrm{C}$, and $\mathrm{E}$, all orally administ d. The patients were then randomly assigned t one of five tre ment arms: arm 1, MPA $500 \mathrm{mg} /$ day or eges ol acetate $320 \mathrm{mg} /$ day; arm 2, oral supplementati with osape aenoic acid; arm 3, L-carnitine $4 \mathrm{~g} /$ day arm thalı ride $200 \mathrm{mg} / \mathrm{day}$; or arm 5, a combinatio of creand The treatment duration was four months. Analy s of variance showed a significant difference between the treament arms, and post hoc analysis showed the superiority of the combination arm (arm 5) over the others for all primary endpoints.

Subsequently, Mantovani et $\mathrm{al}^{60}$ carried out a randomized Phase III study to assess the efficacy of a combination including carnitine and celecoxib \pm megestrol acetate for the treatment of CACS. Analysis of changes from baseline showed that lean body mass as well as physical performance increased significantly in both arms. No significant difference was found between the treatment arms, and treatment was well tolerated. These results suggest that this two-drug combination may be a feasible, effective, and safe approach for CACS in clinical practice.

Macciò et $\mathrm{al}^{61}$ performed a randomized Phase III study in a large selected population of patients with advanced gynecological cancer to assess the safety and efficacy of a multitargeted approach including megestrol acetate, celecoxib, antioxidants (carboxycysteine and lipoic acid), and L-carnitine versus megestrol acetate alone as standard treatment for CACS. These drugs were selected on the basis of monotherapy studies published by Mantovani et al for their ability to target the inflammation, oxidative stress, and metabolic impairment, which are mainly involved in the pathogenesis of symptoms and impaired quality of life in patients with CACS. ${ }^{13,14,34,62}$ The combination treatment arm was found to be more effect ethas negestrol acetate alone in improving lean body ass, restin energy expenditure, fatigue, and global a ality or fe. Mo eover, serum markers of inflammation $2-6$ an C-rea ve protein) and oxidative stress decrea $d$ s. cantly $n$ the combination arm, but did not ch in the a ceiving megestrol acetate alone. Of note in this udy, the gain in lean body mass and global im covment in qu ity of life and subjective symptoms, such a fatigue, hich were observed in the combination therapy a were a sociated with a decrease in the inflammationbasea ogow Prognostic Score. Therefore, the efficacy of anbined treatment in terms of modulation of the inflammatory response associated with improvement in the primary endpoints confirms our hypothesis that the main symptoms of CACS in patients with advanced cancer are driven by systemic inflammation. Moreover, the efficacy of the combination treatment was associated with a significant increase in leptin levels, which may reflect amelioration of metabolic and energy efficiency, as characterized by a reduced resting energy expenditure and an attenuated inflammatory response. These results suggest that monitoring of leptin levels during treatment for CACS can be a useful and relevant parameter of metabolic response.

A multitargeted approach to CACS should be undertaken within the context of the "best supportive care", which includes optimal management of symptoms and careful psychosocial counseling.

\section{Conclusion}

Although various treatments for CACS have been tested, from the results presented here, we can speculate that a single therapy may not be completely successful. Most trials with synthetic progestagens, although currently the only drugs approved for treatment of CACS in Europe, 
have not been shown to improve lean body mass and functional activity, nor have they been shown to improve global quality of life. Further, their significant adverse effects should be taken into account. Among the effective agents, corticosteroids may be useful for their rapid beneficial effects on mood and sense of well-being. However, because of their side effects, short-term and/or alternating administration is recommended. Among the drugs with confirmed clinical efficacy, COX-2 inhibitors and anabolic agents, such as oxandrolone, are well placed to achieve good results. Investigational drugs with potential clinical effectiveness yet to be demonstrated include ghrelin and ghrelin mimetics, SARMs, and drugs targeting inflammatory cytokines, such as OHR/AVR 118 and other anti IL-6 antibodies. Other drugs under investigation include the myostatin inhibitors and $\beta$-adrenoceptor agonists, such APD209 and MT-102.

Considering the complex clinical picture and multifactorial pathogenesis of CACS, we believe that the clinical management of this condition requires a multidisciplinary and multitargeted approach. The recent randomized Phase III clinical trial of five different treatments ${ }^{59}$ should be considered as a template for future approaches. The clearly defined and appropriate endpoints used in that study should also be a reference for future trials, with primary endpoints includ lean body mass, resting energy expenditure and fatigue, an secondary endpoints including muscle strengtb an rexia physical activity levels, quality of life, survi d, and levels of proinflammatory cytokines. ${ }^{35}$

Finally, considering that cachexia starting with precachexia, moving through lifferent stages into overt clinical cachexia, and hally to advan d or refractory cachexia, and that not a vatie sill progress through the complete spectrum an st therapies at the earliest stages of cac'cxia, ssibly ne precachexia stage, with the aim of pre nti sorenying the development of overt cachexia, to obtan the best possible clinical outcome for patients.

\section{Disclosure}

The authors report no conflicts of interest in this work.

\section{References}

1. Delano MJ, Moldawer LL. The origins of cachexia in acute and chronic inflammatory diseases. Nutr Clin Pract. 2006;21(1):68-81.

2. Vigano A, Del Fabbro E, Bruera E, Borod M. The cachexia clinic: from staging to managing nutritional and functional problems in advanced cancer patients. Crit Rev Oncog. 2012;17(3):293-303.

3. Evans WJ, Morley JE, Argiles J, et al. Cachexia: a new definition. Clin Nutr. 2008;27(6):793-799.
4. Argiles JM, Busquets S, Toledo M, Lopez-Soriano FJ. The role of cytokines in cancer cachexia. Curr Opin Support Palliat Care. 2009;3(4):263-268.

5. McDonald N. Cancer cachexia and targeting chronic inflammation: a unified approach to cancer treatment and palliative/supportive care. J Support Oncol. 2007;5(4):157-162.

6. Oldenburg HS, Rogy MA, Lazarus DD, et al. Cachexia and the acutephase protein response in inflammation are regulated by interleukin-6. Eur J Immunol. 1993;23(8):1889-1894.

7. Lokireddy S, Wijesoma IW, Bonala S, et al. Myostatin is a novel tumoral factor that induces cancer cachexia. Biochem J. 2012;446(1): 23-36.

8. Melstrom LG, Melstrom KA Jr, Ding XZ, Adrian TE. Mechanisms of skeletal muscle degradation and its therapy in cancer cachexia. Histol Histopathol. 2007;22(7):805-814.

9. Dodson S, Baracos VE, Jatoi A, et al. Muscle wasting in cancer cachexia: clinical implications, diagnosis, and emerging treatment strategies. Annu Rev Med. 2012;62:265-279.

10. Gautron L, Laye S. Neurobiology of inflammation-associated anorexia. Front Neurosci. 2009;3:59.

11. Thaler JP, Choi SJ, Schy $z$ MW, Visse BE. Hypothalamic inflammation and energy meostasis: $r$ plving the paradox. Front Neuroendocrinol. 201091(1) 84.

12. Tisdale MJ. Mechar ms of canc ach a. Physiol Rev. 2009;89(2): 381-410.

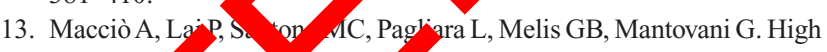
serum ley of solub "L-2 re ptor, cytokines, and $\mathrm{C}$ reactive protein correla impairme cell response in patients with advanced epithorial ova n cancer. oynecol Oncol. 1998;69(3):248-252.

14. Mvani G, M ciò A, Lai P, Massa E, Ghiani M, Santona MC. Cytokine involven.ent in cancer anorexia/cachexia: role of megestrol acet and medroxyprogesterone acetate on cytokine downregulation hd improvement of clinical symptoms. Crit Rev Oncog. 98;90 9-106.

15. Morrs on SD. Partition of energy expenditure between host and tumor. - nycer Res. 1971;31(2):98-107.

16. Straub RH, Cutolo M, Buttgereit F, Pongratz G. Energy regulation and neuroendocrine-immune control in chronic inflammatory diseases. J Intern Med. 2010;267(6):543-560.

17. Del Fabbro E. More is better: a multimodality approach to cancer cachexia. Oncologist. 2010;15(2):119-121.

18. Fearon KC. Cancer cachexia: developing multimodal therapy for a multidimensional problem. Eur J Cancer. 2008;44(8):1124-1132.

19. Fearon K, Strasser F, Anker SD, et al. Definition and classification of cancer cachexia: an international consensus. Lancet Oncol. 2011; 12(5):489-495.

20. Madeddu C, Macciò A, Panzone F, Tanca FM, Mantovani G. Medroxyprogesterone acetate in the management of cancer cachexia. Expert Opin Pharmacother. 2009;10(8):1359-1366.

21. Pascual Lopez A, Roque i Figuls M, Urrutia Cuchi G, et al. Systematic review of megestrol acetate in the treatment of anorexia-cachexia syndrome. J Pain Symptom Manage. 2004;27(4):360-369.

22. Mantovani G, Macciò A, Massa E, Madeddu C. Managing cancer-related anorexia/cachexia. Drugs. 2001;61(4):499-514.

23. Simons JP, Aaronson NK, Vansteenkiste JF, et al. Effects of medroxyprogesterone acetate on appetite, weight, and quality of life in advancedstage non-hormone-sensitive cancer: a placebo-controlled multicenter study. J Clin Oncol. 1996;14(4):1077-1084.

24. Berenstein EG, Ortiz Z. Megestrol acetate for the treatment of anorexia-cachexia syndrome. Cochrane Database Syst Rev. 2005;2: CD004310.

25. Femia RA, Goyette RE. The science of megestrol acetate delivery: potential to improve outcomes in cachexia. BioDrugs. 2005;19(3): 179-187.

26. Bruera E, Roca E, Cedaro L, Carraro S, Chacon R. Action of oral methylprednisolone in terminal cancer patients: a prospective randomized double-blind study. Cancer Treat Rep. 1985;69(7-8):751-754. 
27. Willox JC, Corr J, Shaw J, Richardson M, Calman KC, Drennan M. Prednisolone as an appetite stimulant in patients with cancer. $\mathrm{Br}$ Med $J$ (Clin Res Ed). 1984;288(6410):27.

28. Moertel CG, Schutt AJ, Reitemeier RJ, Hahn RG. Corticosteroid therapy of preterminal gastrointestinal cancer. Cancer. 1974;33(6): 1607-1609.

29. Della Cuna GR, Pellegrini A, Piazzi M. Effect of methylprednisolone sodium succinate on quality of life in preterminal cancer patients: a placebo-controlled, multicenter study. The Methylprednisolone Preterminal Cancer Study Group. Eur J Cancer Clin Oncol. 1989;25(12):1817-1821.

30. Popiela T, Lucchi R, Giongo F. Methylprednisolone as palliative therapy for female terminal cancer patients. The Methylprednisolone Female Preterminal Cancer Study Group. Eur J Cancer Clin Oncol. 1989;25(12):1823-1829.

31. Lesser G, Case D, Ottery F. A phase II randomized study comparing the effects of oxandrolone (Ox) and megestrol acetate (Meg) on lean body mass (LBM), weight (wt) and quality of life (QOL) in patients with solid tumors and weight loss receiving chemotherapy. Proc Am Soc Clin Oncol. 2008;26(Suppl 15):505s.

32. Gullett NP, Hebbar G, Ziegler TR. Update on clinical trials of growth factors and anabolic steroids in cachexia and wasting. Am J Clin Nutr. 2010;91(4):1143S-1147S

33. Lai V, George J, Richey L, et al. Results of a pilot study of the effects of celecoxib on cancer cachexia in patients with cancer of the head, neck, and gastrointestinal tract. Head Neck. 2008;30(1):67-74.

34. Mantovani G, Macciò A, Madeddu C, et al. Phase II nonrandomized study of the efficacy and safety of COX-2 inhibitor celecoxib on patients with cancer cachexia. J Mol Med (Berl). 2010;88(1):85-92.

35. Murphy KT, Chee A, Gleeson BG, et al. Antibody-directed myostatin inhibition enhances muscle mass and function in tumorbearing mice. Am J Physiol Regul Integr Comp Physiol. 2011;301(3): $\mathrm{R} 716-\mathrm{R} 726$

36. Gordon JN, Trebble TM, Ellis RD, Duncan HD, Johns T, Goggin PM. Thalidomide in the treatment of cancer cachexia: a randomised plad controlled trial. Gut. 2005;54(4):540-545.

37. Reid J, Mills M, Cantwell M, Cardwell CR, Murray LJ, Dannelly M Thalidomide for managing cancer cachexia. Cochran atab e Syst Rev. 2012;4:CD008664.

38. Del Fabbro E, Dev R, Hui D, Palmer L, Bruera on appetite and other symptoms in patient and cachexia: a double-blind placebo-co 2013;31(10):1271-1276.

39. DeBoer MD. Emergence of ghre as a treatm for cachexia syndromes. Nutrition. 2008;24

40. Garcia J. A phase II randomizea, aceb ontrolled, double-blind study of the efficacy and safety of RC- 1 (RC) for treatment of cancer cachexia. J Clin Oncol $-007,5$ Sup 4 bo 9133.

41. Garcia JM, Friend Allen Therape cetential of anamorelin, a novel, oral ghrelin met, sir with cancer-related cachexia: a multicenter, randomiz double-blind, crossover, pilot study. Support Care Cancer. 2013;21(1): -137.

42. Dallmann R, Weyermann , Anklin C, et al. The orally active melanocortin-4 receptor antagonist BL-6020/979: a promising candidate for the treatment of cancer cachexia. J Cachexia Sarcopenia Muscle. 2011;2(3):163-174.

43. Rigas J, Schuster M, Orlov S, et al. Efect of ALD518, a humanized anti-IL-6 antibody, on lean body mass loss and symptoms in patients with advanced non-small cell lung cancer (NSCLC): results of a phase II randomized, double-blind safety and efficacy trial. J Clin Oncol. 2010;28:15 Suppl:Abstr 7622.

44. Chasen M, Hirschman SZ, Bhargava R. Phase II study of the novel peptide-nucleic acid OHR118 in the management of cancer-related anorexia/cachexia. J Am Med Dir Assoc. 2011;12(1):62-67.

45. Bayliss TJ, Smith JT, Schuster M, Dragnev KH, Rigas JR. A humanized anti-IL-6 antibody (ALD518) in non-small cell lung cancer. Expert Opin Biol Ther. 2011;11(12):1663-1668.
46. Prado CM, Bekaii-Saab T, Doyle LA, et al. Skeletal muscle anabolism is a side effect of therapy with the MEK inhibitor: selumetinib in patients with cholangiocarcinoma. Br J Cancer. 2012;106(10):1583-1586.

47. Zhou X, Wang JL, Lu J, et al. Reversal of cancer cachexia and muscle wasting by ActRIIB antagonism leads to prolonged survival. Cell. 2010;142(4):531-543.

48. US National Library of Medicine. ClinicalTrials.gov. A Phase 2 study of LY2495655 in participants with pancreatic cancer. Available from: http://clinicaltrials.gov/show/NCT01505530. Accessed May 19, 2013.

49. Busquets S, Figueras MT, Fuster G, et al. Anticachectic effects of formoterol: a drug for potential treatment of muscle wasting. Cancer Res. 2004;64(18):6725-6731.

50. von Haehling S, Stepney R, Anker SD. Advances in understanding and treating cardiac cachexia: highlights from the 5th Cachexia Conference. Int J Cardiol. 2010;144(3):347-349.

51. Stewart Coats AJ, Srinivasan V, Surendran J, et al. The ACT-ONE trial, a multicentre, randomised, double-blind, placebo-controlled, dosefinding study of the anabolic/catabolic transforming agent, MT-102 in subjects with cachexia related to stage III and IV non-small cell lung cancer and colorectal cancer: design. J Cachexia Sarcopenia Muscle. 2011;2(4):201-207

52. Jatoi A, Windschitl HE, oprinzi CL, t al. Dronabinol versus megestrol acetate ver con nation th apy for cancer-associated anorexia: a North C iral Cancer atm Group study. J Clin Oncol. 2002;20(2):567

53. Jatoi A, Roymano prinzi $C$, et al. An eicosapentaenoic acid suppleme versus a restrol cetate versus both for patients with cancer a iated wasti North Central Cancer Treatment Group and Rational ancer Instrute of Canada collaborative effort. $J$ Clin 2004;22 :2469-2476.

McMillan DC, Wismore SJ, Fearon KC, O'Gorman P, Wright CE, McArdle S. A prospective randomized study of megestrol acetate and ibupro $\mathrm{n}$ in gastrointestinal cancer patients with weight loss. $\mathrm{Br}$ Cancer 999;79(3-4):495-500.

55. Cermetti LC, Navigante AH, Peluffo GD, et al. Effects of celecoxib, droxyprogesterone, and dietary intervention on systemic syndromes in patients with advanced lung adenocarcinoma: a pilot study. $J$ Pain Symptom Manage. 2004;27(1):85-95.

56. Cerchietti LC, Navigante AH, Castro MA. Effects of eicosapentaenoic and docosahexaenoic n-3 fatty acids from fish oil and preferential Cox-2 inhibition on systemic syndromes in patients with advanced lung cancer. Nutr Cancer. 2007;59(1):14-20.

57. Lundholm K, Daneryd P, Bosaeus I, Korner U, Lindholm E. Palliative nutritional intervention in addition to cyclooxygenase and erythropoietin treatment for patients with malignant disease: effects on survival, metabolism, and function. Cancer. 2004;100(9):1967-1977.

58. Mantovani G, Macciò A, Madeddu C, et al. A phase II study with antioxidants, both in the diet and supplemented, pharmaconutritional support, progestagen, and anti-cyclooxygenase-2 showing efficacy and safety in patients with cancer-related anorexia/cachexia and oxidative stress. Cancer Epidemiol Biomarkers Prev. 2006;15(5):1030-1034.

59. Mantovani G, Macciò A, Madeddu C, et al. Randomized phase III clinical trial of five different arms of treatment in 332 patients with cancer cachexia. Oncologist. 2010;15(2):200-211.

60. Madeddu C, Dessi M, Panzone F, et al. Randomized phase III clinical trial of a combined treatment with carnitine + celecoxib $+/-$ megestrol acetate for patients with cancer-related anorexia/cachexia syndrome. Clin Nutr. 2012;31(2):176-182.

61. Macciò A, Madeddu C, Gramignano G, et al. A randomized phase III clinical trial of a combined treatment for cachexia in patients with gynecological cancers: evaluating the impact on metabolic and inflammatory profiles and quality of life. Gynecol Oncol. 2012;124(3):417-425.

62. Mantovani G, Macciò A, Madeddu C, et al. The impact of different antioxidant agents alone or in combination on reactive oxygen species, antioxidant enzymes and cytokines in a series of advanced cancer patients at different sites: correlation with disease progression. Free Radic Res. 2003;37(2):213-223. 


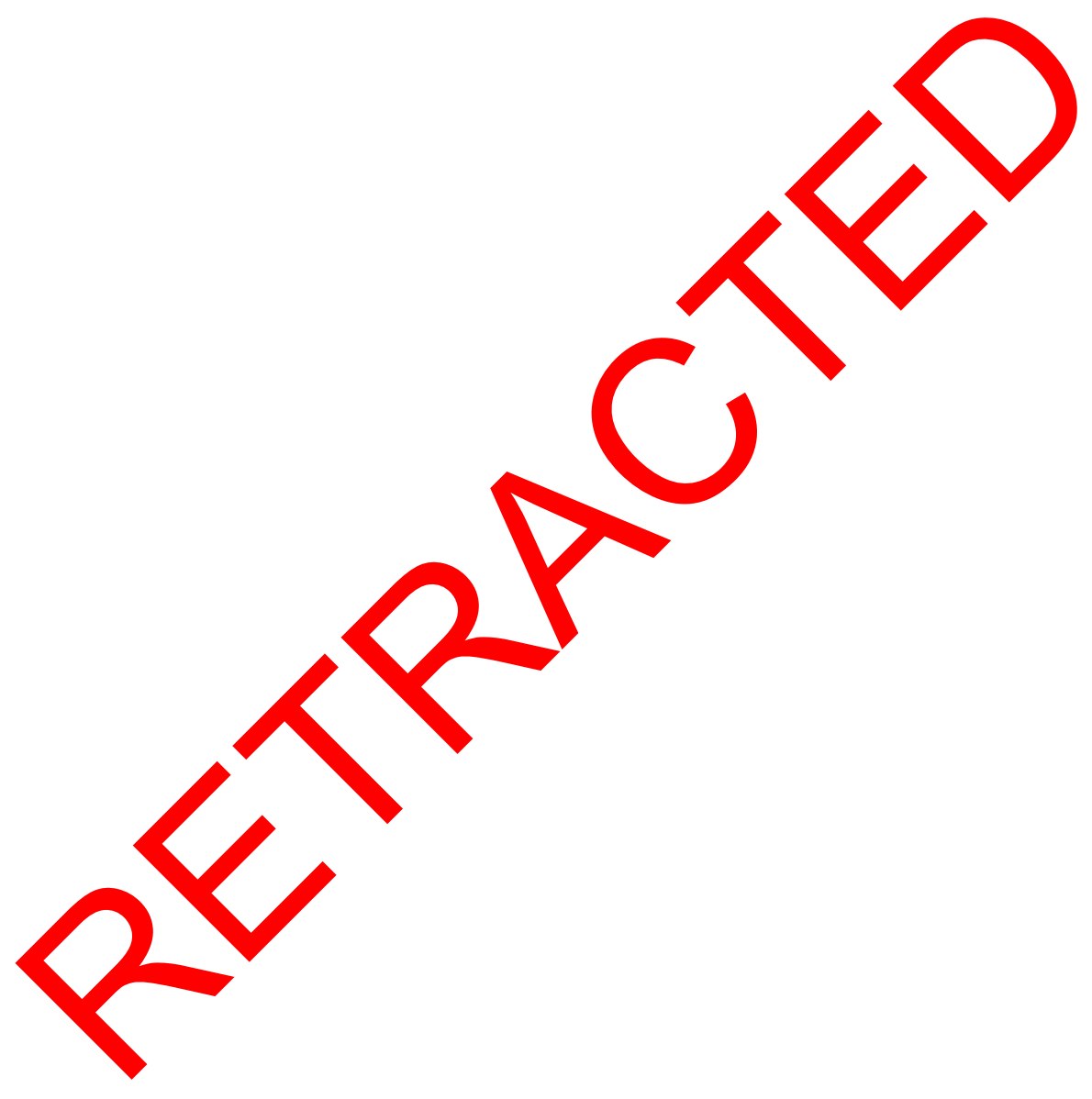

Drug Design, Development and Therapy

Dovepress

\section{Publish your work in this journal}

Drug Design, Development and Therapy is an international, peerreviewed open-access journal that spans the spectrum of drug design and development through to clinical applications. Clinical outcomes, patient safety, and programs for the development and effective, safe, and sustained use of medicines are a feature of the journal, which

has also been accepted for indexing on PubMed Central. The manuscript management system is completely online and includes a very quick and fair peer-review system, which is all easy to use. Visit http://www.dovepress.com/testimonials.php to read real quotes from published authors.

Submit your manuscript here: http://www.dovepress.com/drug-design-development-and-therapy-journal 